Pacific Journal of Mathematics

ON THE RELATIVIZATION OF CHAIN TOPOLOGIES 


\title{
ON THE RELATIVIZATION OF CHAIN TOPOLOGIES
}

\author{
MARCEL ERné
}

The intrinsic topology $\Im_{\lessgtr}$ of a chain $(X, \leqq)$ induces on any subchain $Y \subset X$ the relative topology $\mathfrak{\Im}_{\leq} \mid Y$. On the other hand, any such subchain $Y$ is endowed with its own intrinsic topology $\mathfrak{\Im}_{\leq \mid Y}$. We establish several necessary and sufficient conditions under which both topologies coincide, by suitably weakening the properties of convexity (Lemma 2), order-density (Theorem 3) and subcompleteness (Theorem 4), respectively. Another necessary and sufficient condition for the equation $\mathfrak{\Im}_{\leq}\left|Y=\mathfrak{\Im}_{\leq}\right| Y$, formulated in terms of cuts, is given in Theorem 2. Besides other related results, we find a purely order-theoretical characterization of those subchains which are compact (Lemma 1) or connected (Corollary 2), respectively, in the intrinsic topology of the entire chain. As a simple consequence of Theorem 4, we obtain the wellknown result that the intrinsic topology of a chain can be obtained by relativization from the intrinsic topology of the normal completion (Corollary 9). We conclude with several applications to the Euclidean topology on $R$.

As usual, by a chain, we mean a set $X$ together with a linear (i.e., total) ordering $\leqq$. For the sake of notational brevity, it appears useful to adjoin two new elements $-\infty$ and $\infty$ such that $-\infty<$ $x<\infty$ for all $x \in X$. Each set of the form

$$
] u, v[:=\{x \in X: u<x<v\} \quad(u \in X \cup\{-\infty\}, v \in X \cup\{\infty\})
$$

is called an open interval. The "one-sided infinite" open intervals

and

$$
] u, \infty[=\{x \in X: u<x\} \quad(u \in X)
$$

$$
]-\infty, v[=\{x \in X: x<v\} \quad(v \in X)
$$

are also referred to as open rays. They form a subbase (and the open intervals form a base) for the open sets in the so-called intrinsic topology (or interval topology or order topology, cf. [1], [2], [3], [4]) of the chain $(X, \leqq)$. This topology will be denoted by $\Im_{\leqq}$. Contrary to Birkhoff's definition of topologies in terms of closed sets, we mean by a topology the system of open sets: Thus $\Im_{\leqq}$consists of all set-unions formed by open intervals.

The set-complements of the open rays are the so-called closed rays

$$
]-\infty, u]:=\{x \in X: x \leqq u\} \quad(u \in X)
$$


and

$$
[v, \infty[:=\{x \in X: v \leqq x\} \quad(v \in X) .
$$

Each closed ray and each set of the form

$$
[u, v]:=\{x \in X: u \leqq x \leqq v\} \quad(u, v \in X)
$$

is called a closed interval. Any such interval is, in fact, closed in the intrinsic topology.

Each subset $Y$ of $X$, together with the induced ordering $\leqq \mid Y$, is again a chain and will be referred to as a subchain of $X$. (Precisely, one should distinguish between the subchain $(Y, \leqq \mid Y)$ and the subset $Y$, but since we always assume a fixed linear ordering $\leqq$ to be given, no confusion is likely to arise if the set $Y$ itself is referred to as a subchain). For any such subchain, we may consider its own intrinsic topology $\widetilde{\Im}_{\leqq}$. On the other hand, the intrinsic topology $\mathfrak{\Im}_{\S}$ of the entire chain $X$ induces a relative topology

$$
\mathfrak{\Im}_{\leqq} \mid Y:=\left\{T \cap Y: T \in \mathfrak{\Im}_{\leqq}\right\}
$$

on Y. Obviously, the relative topology $\mathfrak{\Im}_{S_{1}} \mid Y$ is always finer than the intrinsic topology $\left.\mathfrak{\Im}_{\leqq}\right|_{Y}$. It is a natural and for several applications important question under what circumstances both topologies, $\mathfrak{\Im}_{\leqq}$and $\mathfrak{\Im}_{\leqq} \mid Y$, coincide. Subchains $Y$ with this property will be called order-compatible.

By $Y^{i}$ and $Y^{\dagger}$ we denote the set of all lower and upper bounds of $Y$, respectively. $Y$ is convex if for all $x, z \in Y$ and all $y \in X, x<$ $y<z$ implies $y \in Y$. It is easy to see that a subset $Y$ of a chain $X$ is convex if and only if

$$
Y \cup Y^{\downarrow} \cup Y^{\dagger}=X
$$

A subset $Y$ is (order-) dense in $X$ if for all $x, z \in X$ with $x<z$, there exists an element $y \in Y$ with $x<y<z$.

A third order-theoretical property will be of importance for our considerations: A nonempty subset $Y$ of $X$ is subcomplete if every nonempty subset $Z$ of $Y$ has a join and a meet in $X$, and these joins and meets are contained in $Y$. For this definition, we do not require the entire chain $X$ to be complete. For example, the closed interval $[0,1]$ is a subcomplete subchain of the noncomplete chain $\boldsymbol{R}$ of all real numbers. A subcomplete subchain is always a complete chain for itself but a complete subchain need not be subcomplete: See Example 1. It is well-known that a chain is complete if and only if it is compact in its intrinsic topology (cf. [2, p. 242] and [3]). A slight modification gives the following improvement: 
LEMMA 1. A subchain $Y$ of a chain $X$ is subcomplete if and only if it is compact in the intrinsic topology of the entire chain $X$.

In other words, for a subchain to be complete for itself, it is necessary and sufficient that the space $\left(Y, \mathfrak{\Im}_{\leqq I Y}\right)$ be compact, whereas $Y$ is subcomplete if and only if the relativization $\left(Y, \mathfrak{\Im}_{\leq} \mid Y\right)$ is compact. Thus for order-compatible subchains, completeness and subcompleteness are equivalent properties.

Now let us study, in greater detail, the question which subchains are order-compatible. First, we observe that each of the following conditions is sufficient for the equality of $\widetilde{\Im}_{\leqq}$and $\widetilde{\Im}_{\leqq} \mid Y$ :

(1) $Y$ is convex

(2) $Y$ is order-dense in $X$

(3) $Y$ is subcomplete

(4) $Y$ is a finite union of closed intervals

(5) $Y$ is a connected subset of the space $\left(X, \mathfrak{\Im}_{\subseteq}\right)$

(6) $Y$ is a compact subset of the space $\left(X, \Im_{\leqq}\right)$.

The sufficiency of (1) will follow from Lemma 2, that of (2) and (4) from Theorem 3, and that of (3) from Theorem 4. Furthermore, a connected subchain is always convex (see Corollary 2), and (3) is equivalent with (6), on account of Lemma 1.

Simple counterexamples show that none of the previously listed properties is necessary for order-compatibility. On the other hand, none of the following conditions is even sufficient:

(7) $Y$ is a complete chain for itself

(8) $Y$ is a union of an open and a closed interval

(9) $Y$ is dense-in-itself

(10) $Y$ is topologically dense in $\left(X, \mathfrak{\Im}_{\leq}\right)$

(11) $Y$ is open in $\left(X, \Im_{\leqq}\right)$

(12) $Y$ is a union of two open intervals

(13) $Y$ is bounded and closed in $\left(X, \mathfrak{\Im}_{5}\right)$.

EXAMPLE 1. $Y:=[0,1] \cup] 2,3]$ is a complete subchain of the complete chain $X=[0,1] \cup[2,3] \subset[0,3] \subset \boldsymbol{R}$ but $Y$ is not subcomplete. Furthermore, $Y$ is a disjoint union of an open and a closed interval but also a union of two open intervals and therefore open in $\mathfrak{\Im}_{\Xi}: Y=$ (]$-\infty, 2[\cap X) \cup(] 2, \infty[\cap X)$. Finally, $Y$ is dense-in-itself as well as topologically dense in $\left(X, \mathfrak{\Im}_{\S}\right)$ but not order-dense in $X$. The interval $[0,1] \subset Y$ is open in $\mathfrak{\Im}_{\leqq} \mid Y$ but not in $\mathfrak{\Im}_{\leqq 1 Y}$.

The complete chain $X$ in the preceding example cannot serve as a counterexample disproving the sufficiency of (13) since every closed subset of any complete chain is order-compatible (see Corollary 5). For this purpose, consider 
EXAMPLE 2. The chain $Y=\left\{x \in \boldsymbol{Q}: 2<x^{2} \leqq 4\right.$ or $\left.x=0\right\}$ is bounded and closed in $\left(\boldsymbol{Q}, \mathfrak{\Im}_{\leqq}\right)$but $\{0\} \in \mathfrak{\Im}_{\leqq} \mid Y,\{0\} \notin \mathfrak{\Im}_{\leqq \mid Y}$. Note that $Y$ is not compact in $\left(\boldsymbol{Q}, \mathfrak{\Im}_{\leq}\right)$. Hence the Heine-Borel-Lebesgue theorem fails to be true in the chain $\boldsymbol{Q}$. It can be shown that for a chain to be conditionally complete, it is necessary and sufficient that every closedbounded subchain be compact.

Under certain additional assumptions, one can prove that closed or open subchains are order-compatible: See Corollaries 5 and 6 .

As mentioned before, neither convexity nor density nor subcompleteness is necessary for order-compatibility. One necessary and sufficient condition has been found by R. Alo and O. Frink [1]:

THeOREM 1. A subchain $Y$ of the chain $X$ is order-compatible if and only if for all $y \in Y$ and all $x \in X \backslash Y$, at least one of the following conditions holds:

(a) $] y, x[\cap Y$ is nonempty.

(b) $y<x$ and $[x, \infty[\cap Y$ has a least element.

(c) $] x, y[\cap Y$ is nonempty.

(d) $x<y$ and] $-\infty, x] \cap Y$ has a greatest element.

This theorem will be an easy consequence of Corollary 4 .

A nearly obvious (but technically less handsome) necessary and sufficient condition is given in

LEMMA 2. A subchain $Y$ of $X$ is order-compatible if and only if for all $x \in X$ which are neither elements nor lower nor upper bounds of $Y$, (that is, for all elements $x \in X \backslash\left(Y \cup Y^{\downarrow} \cup Y^{\uparrow}\right)$ ), the sets $]-\infty, x] \cap Y$ and $\left[x, \infty\left[\cap Y\right.\right.$ are open (and closed) in $\mathfrak{J}_{\leqq \mid Y}$.

Proof. The necessity of this condition is clear. To prove sufficiency, we observe that the system

$$
\{\quad-\infty, x[\cap Y: x \in X\} \cup\{] x, \infty[\cap Y: x \in X\}
$$

is a subbase for the relative topology $\Im_{\leqq} \mid Y$. Hence it is enough to show that for all $x \in X$, the sets $U:=]-\infty, x[\cap Y$ and $V:=$ ]$x, \infty\left[\cap Y\right.$ are open in $\Im_{\leqq \mid Y}$. For $x \in Y$, this is clear since then $U$ and $V$ are open rays in the subchain $Y$. For $x \in Y^{\downarrow} \backslash Y$, we have $U=\varnothing$ and $V=Y$, and for $x \in Y^{\uparrow} \backslash Y, U=Y$ and $V=\varnothing$. Finally, for $x \in$ $\left.X \backslash\left(Y \cup Y^{i} \cup Y^{\uparrow}\right), U=\right]-\infty, x[\cap Y$ and $V=] x, \infty[\cap Y$ are open in $\Im_{\leq \mid Y}$ on account of the assumption made in Lemma 2.

Since for any convex subchain $Y$, the set $X \backslash\left(Y \cup Y^{+} \cup Y^{-}\right)$is 
empty, we infer immediately

Corollary 1. Every convex subchain of $X$ is order-comptaible.

Corollary 2. For a subchain $Y$ of $X$, the following three conditions are equivalent:

(a) $Y$ is connected in the intrinsic topology of $X$.

(b) $Y$ is order-compatible and connected in its own intrinsic topology.

(c) $Y$ is convex, conditionally complete and dense-in-itself.

Proof. If $Y$ is not convex then there are elements $y, z \in Y$ and $x \in X \backslash Y$ with $y<x<z$. But then $U:=]-\infty, x] \cap Y=]-\infty, x[\cap Y$ and $V:=[x, \infty[\cap Y=] x, \infty[\cap Y$ are pairwise complementary nonempty subsets of $Y$ which are both open and closed in $\Im_{\leqq} \mid Y$. Hence $Y$ cannot be connected in the space $\left(X, \Im_{\leqq}\right)$. Furthermore, it is well-known (cf. [2, p. 243] or [4, p. 68]) that a chain is connected in its intrinsic topology if and only if it is conditionally complete and dense-in-itself. Thus, from Corollary 1, we infer the equivalence of (a), (b) and (c).

A segment or ideal of the chain $X$ is a subset $S$ such that for all $x \in X$ and all $y \in S, x \leqq y$ implies $x \in S$. A cut or closed ideal (cf. [2, p. 127]) is a subset $C$ of $X$ such that $C=C^{\dagger \downarrow}$. Each closed ray of the form $]-\infty, x]$ is a cut, called the principal cut (or principal $i d e a l$ ) generated by $x$. Without proof, we summarize several alternative characterizations of cuts:

LEMma 3. For any nonempty subset $C$ of the chain $X$, the following conditions are all equivalent:

(a) $C$ is a cut of $X$.

(b) $C$ is an ideal and closed in the intrinsic topology of $X$.

(c) $C=D^{\downarrow}$ for some subset $D$ of $X$.

(d) $C$ is an intersection of principal cuts.

(e) $C$ is an ideal, and if $C$ has a least upper bound $y$ then $y$ is the greatest element of $C$.

(f) $x<y$ for all $x \in C$ and all $y \in X \backslash C$, and if $C$ has no greatest element then $X \backslash C$ has no least element.

(g) $C$ is a principal cut, or $C^{\uparrow}=X \backslash C$ has no least element.

We notice that for a chain without least element, the empty set is a cut, while for chains with least element, every cut is nonempty (containing the least element). The collection $\tilde{X}$ of all cuts in $X$ is closed under arbitrary intersections and therefore a complete lattice, linearly ordered by set inclusion. $\tilde{X}$ is called the normal completion 
or completion by cuts of $X$ (cf. [2, p. 127]).

A dual cut is a subset $D$ of $X$ with $D=D^{\natural \uparrow}$. Now one can prove easily

THEOREM 2. For a subchain $Y$ of $X$, the following three conditions are equivalent:

(a) $Y$ is order-compatible.

(b) For each cut $C$ of $X$, the relativization $C \cap Y$ is empty or a cut of $Y$, and dually.

(c) For each $x \in X \backslash\left(Y \cup Y^{\downarrow} \cup Y^{\dagger}\right)$, the set $\left.]-\infty, x\right] \cap Y$ is a cut of $Y$, and the set $[x, \infty[\cap Y$ is a dual cut of $Y$.

Proof. $\quad(a) \Rightarrow(b)$ : Every cut $C$ of $X$ is an intersection of closed rays: $\left.\left.C=C^{\uparrow \downarrow}=\bigcap\{]-\infty, x\right]: x \in C^{\dagger}\right\}$. Each of the sets $\left.]-\infty, x\right] \cap Y$ is an ideal of $Y$ and closed in the relative topology $\Im_{\leq} \mid Y$ which coincides, by hypothesis, with the intrinsic topology $\mathfrak{J}_{\leqq}$. From Lemma 3, we infer that $]-\infty, x] \cap Y$ is empty or a cut in $Y$. Since the system of all cuts is closed under arbitrary intersections, it follows that $\left.C \cap Y=\bigcap\{]-\infty, x] \cap Y: x \in C^{\dagger}\right\}$ is also empty or a cut in $Y$. For dual cuts, we conclude analogously.

$(b) \Rightarrow(c)$ : Trivial.

(c) $\Rightarrow$ (a): Clear by Lemmas 2 and 3 .

Combining Theorem 2 with the equivalence $(\mathbf{a}) \Leftrightarrow(\mathbf{f})$ in Lemma 3 , we obtain

CoRollary 3. A subchain $Y$ of $X$ is order-compatible if and only if it satisfies the following symmetry condition: For each $x \in X \backslash\left(Y \cup Y^{\dagger} \cup Y^{\uparrow}\right)$, the set $[x, \infty[\cap Y$ has a least element if and only if the set $]-\infty, x] \cap Y$ has a greatest element.

This may be reformulated as

CoROllary 4. For a subchain $Y$ of $X$ to be order-compatible, the following condition is necessary and sufficient: If $x$ is any element of $X$ such that the sets $U:=]-\infty, x] \cap Y$ and $V:=[x, \infty[\cap Y$ are nonempty then $U$ has a greatest element if and only if $V$ has a least element.

Lemma 4. A subchain $Y$ of a conditionally complete chain $X$ is closed in the intrinsic topology of $X$ if and only if for every $x \in X,]-\infty, x] \cap Y$ is empty or has a greatest element, and $] x, \infty] \cap$. $Y$ is empty or has a least element. 
Proof. Suppose $Y$ is closed, and $U:=]-\infty, x] \cap Y \neq \varnothing$. Conditional completeness ensures the existence of a least upper bound $z$ for $U$. If $z$ is in the complement of $Y$ then there is an open interval ] $u$, $v$ [ with $z \in] u, v[\subset X \backslash Y$. The case $u=-\infty$ is impossible since every element of $U$ is a lower bound for $] u, v[$. Thus $u$ is an upper bound of $U$, contradicting the minimality of $z$. Hence $z$ must be the greatest element of $U$. By dual arguments, the existence of a least element for $[x, \infty[\cap Y \neq \varnothing$ can be shown.

Conversely, assume the condition stated in Lemma 4 is fulfilled for some subset $Y \subset X$. We have to show that $X \backslash Y$ is open in the interval topology: Assume $x \in X \backslash Y$ and $x<z$. If $[x, z[\not \subset X \backslash Y$ then there exists $y \in[x, \infty[\cap Y \neq \varnothing$. Let $v$ be the least element of $[x, \infty[\cap Y$. Then we have $x<v$, and $[x, v[\subset X \backslash Y$. Dually, if $x$ is not the least element of $X$, we find $u \in]-\infty, x[\cap Y$ with $] u, x] \subset X \backslash Y$. In any case, there are elements $u \in X \cup\{-\infty\}$ and $v \in X \cup\{\infty\}$ with $x \in$ ]$u, v[\subset X \backslash Y$.

COROLLARY 5. Every closed subset of a conditionally complete chain is order-compatible.

Another possibility to characterize order-compatible subchains is suggested by the trivial fact that on every dense subchain, the intrinsic topology coincides with the relative topology: We say a subset $Y \subset X$ separates two points $x, z \in X$ if there exists a $y \in Y$ with $x<y<z$ or $z<y<x$. Accordingly, $Y$ is (order-) dense in $X$ if and only if $Y$ separates any two different points of $X$. Similarly, $Y$ is convex if and only if $X \backslash Y$ does not separate any pair of distinct points in $Y$. Since density is sufficient but not necessary for ordercompatibility, it appears obvious to weaken the separation property of dense subchains in such a manner that a sufficient and necessary condition for order-compatibility is obtained. Thus we call a subset $Y \subset X$ weakly dense if it separates any point of $Y$ from any point of the set $K(Y)$ consisting of all those $x \in X$ which are neither elements nor lower bounds nor upper bounds of $Y$, nor members of the set

$$
I(Y)=\mathrm{U}\{\quad] y, z[: y, z \in Y,] y, z[\cap Y=\varnothing\}
$$

Thus

$$
K(Y)=X \backslash\left(Y \cup Y^{\downarrow} \cup Y^{\dagger} \cup I(Y)\right) .
$$

For convex subsets, $I(Y)$ and $K(Y)$ are empty while for dense subsets of nonbounded chains, $I(Y)$ is empty and $K(Y)$ is the set-complement of $Y$. Now our third characterization theorem: 
THEOREM 3. A subchain is order-compatible if and only if it is weakly dense.

Proof. Let $Y$ be weakly dense. By Lemma 2 we have to show that for all $x \in X \backslash\left(Y \cup Y_{+} \cup Y^{-}\right)$, the sets $]-\infty, x[\cap Y$ and $] x, \infty[\cap Y$ are open in the interval topology on $Y$ :

Case 1. $x \in I(Y)$ : Then there are elements $y, z \in Y$ with $x \in$ ]$y, z[$ and $] y, z[\cap Y=\varnothing$. Hence $]-\infty, x[\cap Y=]-\infty, z[\cap Y \in$ $\left.\Im_{\leqq \mid Y^{\prime}}\right] x, \infty\left[\cap Y \in \Im_{\leq \mid Y^{\prime}}\right.$.

Case 2. $x \in K(Y)$ : For $z \in] x, \infty[\cap Y$, it follows $x<z, z \in Y$, and since $Y$ is weakly dense, we find $y \in Y$ with $x<y<z$. Thus $z \in] y, \infty[\cap Y \subset] x, \infty[\cap Y$, and $] x, \infty\left[\cap Y\right.$ contains an $\mathfrak{\Im}_{\leqq}-$open neighborhood of each of its points. Consequently, $] x, \infty[\cap Y$ is open in $\mathfrak{\Im}_{\leq 1 X}$.

By dual arguments, we obtain $]-\infty, x\left[\cap Y \in \widetilde{\Im}_{\leqq \mid Y^{\prime}}\right.$ and summarizing, we conclude that the subchain $Y$ is order-compatible.

Conversely, assume each of the sets $] x, \infty[\cap Y$ and $]-\infty, x[\cap Y$ is open in $\mathfrak{\Im}_{\leqq \mid Y}$. We have to separate each element $x \in Y$ from each element $z \in K(Y)$ by some $y \in Y$. Without loss of generality, we may consider the case $x<z$. Assume $] x, z[\cap Y=\varnothing$. By definition of $K(Y), z$ is neither an element nor an upper bound of $Y$. Furthermore ]$-\infty, z[\cap Y=]-\infty, x] \cap Y$ is open in $\Im_{\leqq Y Y}$ whence $x$ must be covered in $Y$ by some $y \in Y$. (Note that $x$ cannot be the greatest element of $Y$ since $z$ is not an upper bound of $Y$.) Hence $x \in]-\infty, z[\cap Y=$ ]$-\infty, x] \cap Y=]-\infty, y[\cap Y$. The assumption $y<z$ leads to the contradiction $y \in] x, z[\cap Y \neq \varnothing$. But on the other hand, $z<y$ implies ]$z, y[\cap Y=\varnothing] x,, y[\cap Y=\varnothing, z \in] x, y[\subset I(Y)$, which is a contradiction to the choice of $z \in K(Y)$. Hence we must have $] x, z[\cap Y \neq \varnothing$, as desired.

LemMa 5. Let $Y$ be an open subset of a dense chain $X$. Then $Y$ separates each point of $Y$ from each point of $X \backslash Y$. In particular, $Y$ is weakly dense.

Proof. Suppose $x \in Y, z \in X \backslash Y$ and $x<z$. Choose an open interval ]$u, v[\subset Y$ with $x \in] u, v[$. We may assume $v \neq \infty$ since $x$ is not the greatest element of $X$. It follows $x<v \leqq z$ and $[x, v[\subset Y$. By density of $X$, there exists $y \in X$ with $x<y<v$, and $[x, v[\subset Y$ implies $y \in Y$. Thus we have separated $x$ and $z$ by a point of $Y$. The case $z<x$ can be treated dually.

Corollary 6. Every open subchain of a dense chain is ordercompatible. 
Another consequence of Theorem 3 is

CoRollary 7. Every dense subset, every convex subset and every finite union of closed intervals is weakly dense and therefore ordercompatible.

This is clear since for convex subsets and finite unions of closed intervals, the set $K(Y)$ is empty.

By suitably weakening the convexity and the density concept, respectively, we have found two necessary and sufficient conditions for order-compatibility. A third one can be obtained by weakening the property of subcompleteness: Let $Y$ be any subchain of $X$ such that whenever a nonempty subset $Z$ of $Y$ has a join (meet) in $Y$ then this is also the join (meet) of $Z$ in $X$. In this case, we say the subchin $Y$ is weakly subcomplete. Clearly, subcompleteness implies weak subcompleteness, but not conversely. Note that $Y$ may be weakly subcomplete in $X$ though neither $X$ nor $Y$ are complete chains (Example: $\boldsymbol{Q} \subset \boldsymbol{R}$ ).

We notice that every chain is weakly subcomplete in its normal completion and this completion is a complete chain.

THEOREM 4. A subchain $Y$ is order-compatible if and only if it is weakly subcomplete.

Proof. Suppose $Y$ is order-compatible, and let $Z$ be a nonempty subset of $Y$ which has a meet $y$ in $Y$. If $y$ is not the meet in $X$ then there is a greater lower bound $z$ of $Z$ which belongs to the complement of $Y$, and it follows $] y, z[\cap Y=\varnothing$.

We show that $z$ is an element of $K(Y): z$ is neither a lower bound of $Y$ (since $y<z$ ) nor an upper bound of $Y$ (since $\varnothing \neq Z \subset Y$ and $z<w$ for all $w \in Z$ ). If $z$ would be a member of $I(Y)$ then there would exist $u, v \in Y$ such that $z \in] u, v[\subset X \backslash Y$, and $v$ would be a lower bound of $Z$ in $Y$ greater than $y$, a contradiction. Hence $z \in$ $X \backslash\left(Y \cup Y^{\downarrow} \cup Y^{\uparrow} \cup I(Y)\right)$. But $y$ and $z$ cannot be separated by $Y$ contradicting Theorem 3. Hence $y$ is also the meet of $Z$ in $X$. Combining this with dual arguments, it follows that $Y$ is weakly subcomplete.

To complete the proof, it suffices to show that any weakly subcomplete subchain $Y$ satisfies the symmetry condition stated in Corollary 4: Suppose $U:=]-\infty, x] \cap Y$ has a greatest element $y$. If $y$ is not the greatest lower bound of $V$ in $Y$ then there is some $z \in Y$ with $y<z \leqq v$ for all $v \in V$, and $] y, x] \cap Y=\varnothing$ implies $x<z, z \in V$, 
whence $z$ is the least element of $V$. On the other hand, if $y$ is the greatest lower bound of $V$ in $Y$ then it is the meet of $V$ in $X$, too. But $x$ is another lower bound of $V$ with $y \leqq x$, so $y=x$ is the least element of $V$.

CoRollaRY 8. Every subcomplete (that is, compact) subchain is order-compatible.

COROLLARY 9. The intrinsic topology of any chain can be obtained by relativization from the intrinsic topology of the normal completion.

CoRollary 10. On every convex, dense, compact, connected, open or closed subchain of $\boldsymbol{R}$, the intrinsic topology is induced by the Euclidean topology on $\boldsymbol{R}$.

CoROLlaRY 11. For any subgroup $G$ of $\boldsymbol{R}$, the intrinsic topology of $G$ is induced by the Euclidean topology on $\boldsymbol{R}$.

This is a consequence of Corollary 10 and the fact that any subgroup $G \neq\{0\}$ of $\boldsymbol{R}$ is either isomorphic to $\boldsymbol{Z}$ (hence closed in $\boldsymbol{R}$ ) or dense in $\boldsymbol{R}$.

\section{REFERENCES}

1. R. Alo and O. Frink, Topologies of chains, Math. Ann., 171 (1967), 239-246.

2. G. Birkhoff, Lattice theory; third ed., sec. print, Amer. Math. Soc. Colloquium Publ., 25, New York, (1973).

3. O. Frink, Topology in lattices, Trans. Amer. Math. Soc., 51 (1942), 569-583.

4. L. A. Steen and J. A. Seebach, Counterexamples in Topology, Holt, Rinehart and Winston, Inc., New York, 1968.

Received October 18, 1978.

UNIVERSTTÄT HANNOVER

WELFENGARTEN 1

D 3000 HANNOVER

Fed. Rep. OF Germany 


\section{PACIFIC JOURNAL OF MATHEMATICS}

\section{EDITORS}

DONALD BABBITT (Managing Editor)

University of California

Los Angeles, California 90024

Hugo RossI

University of Utah

Salt Lake City, UT 84112

C. C. MOORE and ANDrew OGG

University of California

Berkeley, CA 94720
J. DUgundjI

Department of Mathematics University of Southern California Los Angeles, California 90007

R. FinN aNd J. Milgram Stanford University Stanford, California 94305

\section{ASSOCIATE EDITORS}

E. F. BECKENBACH

B. H. NeUMANN

F. WOLF

K. YosHIDA

\section{SUPPORTING INSTITUTIONS}

UNIVERSITY OF BRITISH COLUMBIA CALIFORNIA INSTITUTE OF TECHNOLOGY UNIVERSITY OF CALIFORNIA MONTANA STATE UNIVERSITY UNIVERSITY OF NEVADA, RENO NEW MEXICO STATE UNIVERSITY OREGON STATE UNIVERSITY UNIVERSITY OF OREGON
UNIVERSITY OF SOUTHERN CALIFORNIA STANFORD UNIVERSITY UNIVERSITY OF HAWAII UNIVERSITY OF TOKYO UNIVERSITY OF UTAH WASHINGTON STATE UNIVERSITY UNIVERSITY OF WASHINGTON 


\section{Pacific Journal of Mathematics}

\section{Vol. 84 , No. 1 \\ May, 1979}

Michael James Beeson, Goodman's theorem and beyond ...............

Robert S. Cahn and Michael E. Taylor, Asymptotic behavior of multiplicities

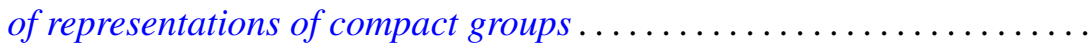

Douglas Michael Campbell and Vikramaditya Singh, Valence properties of



J.-F. Colombeau, Reinhold Meise and Bernard Perrot, A density result in spaces of Silva holomorphic mappings .....................

Marcel Erné, On the relativization of chain topologies .................

Le Baron O. Ferguson, Uniform and $L_{p}$ approximation for generalized

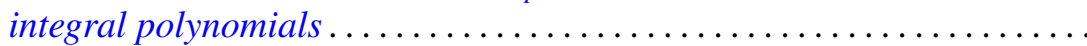

Kenneth R. Goodearl and David E. Handelman, Homogenization of regular rings of bounded index..................................

Friedrich Haslinger, A dual relationship between generalized

Abel-Gončarov bases and certain Pincherle bases .................

Miriam Hausman, Generalization of a theorem of Landau. .

Makoto Hayashi, 2-factorization in finite groups

Robert Marcus, Stochastic diffusion on an unbounded domain ........... 143

Isabel Dotti de Miatello, Extension of actions on Stiefel manifolds....

C. David (Carl) Minda, The hyperbolic metric and coverings of Riemann surfaces...

Somashekhar Amrith Naimpally and Mohan Lal Tikoo, On $T_{1}$-compactifications.

Chia-Ven Pao, Asymptotic stability and nonexistence of global solution for a semilinear parabolic equation ....

Shigeo Segawa, Harmonic majoration of quasibounded type ...

Sze-Kai Tsui and Steve Wright, The splitting of operator algebras ...

Bruce Williams, Hopf invariants, localization and embeddings of Poincaré complexes....

Leslie Wilson, Nonopenness of the set of Thom-Boardman maps ...

Alicia B. Winslow, There are $2^{\mathrm{c}}$ nonhomeomorphic continua in $\beta R^{n}-R^{n}$ 